\title{
ENGAGEMENTY BIENESTAR EN EL PROFESORADO UNIVERSITARIO. HACIA LA DELIMITACIÓN DE INDICADORES EVALUATIVOS
}

\author{
Antonio Bernal Guerrero \\ Macarena Donoso González \\ Universidad de Sevilla
}

RESUMEN: La construcción de la identidad profesional docente se encuentra afectada por el marco de cambios sociales experimentados en nuestro tiempo, asociados a complejos mecanismos de des-institucionalización y de reorganización, produciéndose un considerable desgaste emocional. Hemos realizado una investigación sobre el bienestar docente vinculado al fenómeno denominado engagement, que se presenta como una vía de solución para combatir el malestar docente y promover el bienestar. Dada la escasez de estudios existentes sobre la delimitación de descriptores sustantivos para la evaluación del engagement en el docente universitario, hemos Ilevado a cabo un estudio exploratorio en la Universidad de Sevilla de carácter cualitativo, utilizando el método biográfico-narrativo y la técnica de la entrevista, con la finalidad de avanzar en esta línea formativa. Los resultados del estudio parecen reflejar que hay ciertos factores que inciden en el bienestar: la estabilidad institucional, las condiciones laborales, el reconocimiento social y la mejora de la formación del profesorado. Asimismo, se esboza una serie de indicadores evaluativos relacionada con el ámbito personal: disposición activa hacia el perfeccionamiento, capacidad de establecer relaciones óptimas, perseverancia, vinculación entusiasta con la profesión, y equilibrio emocional reflejado en la conciliación entre los distintos ámbitos de la vida.

PALABRAS CLAVE: Profesión docente, universidad, bienestar, engagement, evaluación del profesor.

\section{ENGAGEMENT AND WELL-BEING UNIVERSITY TEACHER. TOWARDS THE DELIMITATION OF EVALUATIVE INDICATORS}

ABSTRACT: Building a professional teacher identity is affected by the framework of the social changes of our time. These are associated with complex mechanisms of deinstitutionalization and reorganization which 
produce considerable emotional burnout. We have carried out an investigation of teaching well-being linked to the phenomenon called engagement. This is presented as a way to combat teachers' malaise and foster well-being. There is a paucity of studies on the delimiting of substantive descriptors for the evaluation of engagement in university teaching. We have therefore done an exploratory qualitative study in the University of Seville. In doing this, we have used the biographic-narrative method and the interview technique in order to progress in this educational line. The results of the study appear to reflect that there are certain factors which influence well-being: institutional stability, labor conditions, social recognition and the improvement of the teachers' training. Likewise, a series of evaluation indicators connected with the personal level are outlined: an active willingness toward enhancement, a capacity to set up optimal relations, perseverance, an enthusiastic link with the profession and an emotional balance expressed in reconciling different areas of life.

KEYWORDS: Teaching profession, university, well-being, engagement, teacher appraisal.

Recibido: 21/03/2016

Aceptado: 14/07/2016

Correspondencia: Antonio Bernal Guerrero, Departamento de Teoría e Historia de la Educación y Pedagogía Social, Facultad de Ciencias de la Educación, Universidad de Sevilla, C/ Pirotecnia, s/n, 41013, Sevilla. Email: abernal@us.es.

\section{INTRODUCCIÓN}

Por su propio dinamismo reflejamente activado, la conducta humana en el mundo actual adquiere un talante predominantemente contrafáctico, eminentemente contingente e incierto. Estudiosos pertenecientes a diferentes escuelas y tradiciones convergen en un diagnóstico análogo: la progresiva declinación de roles y normas mediante las que se construía el mundo, obligando a los individuos a su construcción, a su autoorganización y autodeterminación (Beck y Beck-Gernsheim, 2003; Dubet, 2006; Giddens, 2000; Touraine, 2005). Nadie escapa a esta necesidad imperiosa de autoconfiguración identitaria. La consideración de la identidad como un efecto más que como una categoría fundacional e inamovible, se abre a un horizonte de múltiples posibilidades y también de riesgos. Los cambios producidos en el mundo del trabajo, cada vez más proteico y elástico, han afectado a la noción de la propia identidad profesional vinculada al desempeño laboral, en la medida en que asociamos lo que se es a lo que se hace, esa "corrosión del carácter" a la que se ha referido Richard Sennett (2000).

La pervivencia de la identidad profesional depende de la reinvención continua. El profesorado se encuentra sometido y afectado por estos cambios ligados al desenclave institucional y a la reorganización espacio-temporal, propios de nuestro tiempo, aunque aparentemente las instituciones, costumbres y usos sociales habi- 
tualmente reconocidos parezcan mostrarse inalterados. Estudiar la profesionalidad del docente implica, por tanto, el reconocimiento del impacto de estas nuevas condiciones sociales; sus identidades serán el producto de identificaciones contingentes, emergidas de las propias reivindicaciones y de atribuciones externas, en circunstancias singulares y desde trayectorias únicas. Este proceso de construcción de la identidad docente se torna esencial para comprender las motivaciones, las satisfacciones y habilidades que dan contenido a su quehacer profesional. No hay identidad profesional desvinculada de la identidad personal (Zembylas, 2003). Junto a la dimensión cognitiva, aflora la emocional como una componente fundamental para entender cómo se construye la identidad (Hargreaves, 2003; Hué, 2008). En este sentido, es de la mayor relevancia la comprensión del ámbito emocional de la práctica docente, no sólo para la intelección de los elementos que conducen al deterioro identitario de la profesión, sino sobre todo para procurar trazar vías esperanzadoras de (re)construcción.

\section{Problemática del docente universitario e incidencia en su nivel de bienestar}

La fuerte vinculación e implicación que requiere la práctica profesional, así como múltiples situaciones educativas y sociales nuevas hacen que el docente se encuentre inmerso en una espiral problemática de difícil control en su desempeño cotidiano. Existe la creencia popular de que el docente universitario no adquiere los mismos niveles de malestar que los de niveles educativos inferiores, presuponiéndose que no tiene problemas de disciplina en las aulas, no tiene presiones de agentes externos e internos, no sufre acoso de sus compañeros y sus dedicaciones presentan una armónica cadencia. Suele considerarse, en suma, que el profesor universitario, centrado eminentemente en su actividad investigadora, no padece los niveles de estrés propios de la profesión en niveles no universitarios, especialmente en la enseñanza secundaria. Sin embargo, la realidad es distinta; el profesorado universitario, en general, carece del reconocimiento social de tiempos pasados y su remuneración se ve mermada en sentido inversamente proporcional al aumento de responsabilidades y al duro escrutinio externo al que se halla continuamente sometido (Barraca, 2010; Bresó, Salanova, Schaufeli y Nogareda, 2005; García Carrasco y Bernal, 2008). El contexto del Espacio Europeo de Educación Superior, junto a las posibilidades inequívocas que suscita y la delimitación de una línea de internacionalización que pensamos que debe proseguir y avanzar, también ofrece aspectos conceptuales, metodológicos y funcionales que no han dejado de incidir en la constatación generalizada del aumento de incertidumbre y ha enfatizado en ciertos aspectos niveles de precariedad y provisionalidad que han coadyuvado al incremento del malestar (Caballero, 2009; Cantón, Valle y Arias, 2008; García y González, 2007). Como han señalado Caballero y Bolívar (2015), los padecimientos del profesorado universitario (desprestigio de la educación, mayor exigencia en la rendición de cuentas, dura lucha y rivalidad para alcanzar grados de estabilidad, frustraciones y estancamiento, etc.) inciden en la conformación de su identidad profesional, afectada por el incremento de la absorción exigida por los nuevos condicionantes y exigencias de su actividad, frecuentemente abocada a un considerable desgaste emocional y a una disminución de su bienestar. 


\section{Afrontamiento físico y emocional del malestar: el engagement como estrategia}

Ante el malestar docente ha habido numerosas estrategias de afrontamiento que han pretendido el control de la situación estresora y sus repercusiones (Amador, Rodríguez, Serrano, Olvera y Martínez, 2014; Caballero, Hederich y Palacio, 2010; Guerrero, 2002; Ibarra, Armenta y Jacobo, 2014; Matud, García y Matud, 2006; Sánchez, March y Ballester, 2015). Contamos con un catálogo de terapias, psicoterapias y programas educativos sobre el afrontamiento del estrés, mediante conductas adaptativas, surgido del auge e impulso que ha tenido la psicología positiva en los últimos años (Augusto-Landa, López-Zafra, Martínez y Pulido-Martos, 2006; Seligman y Csikszentmihalyi, 2000; Vázquez y Hervás, 2009).

En el contexto de las sociedades de riesgo, por recordar la célebre expresión de Ulrich Beck, han surgido consecuentemente nuevas filosofías en las organizaciones que desarrollan políticas de Dirección de Recursos Humanos (DRH) que "se asientan sobre conceptos teorías y aplicaciones psicológicas que pueden mejorar e incrementar la salud, seguridad, motivación y bienestar de los empleados" (Salanova y Schaufeli, 2009: 19). Se ha ido prestando más atención a las diferencias individuales y a los recursos personales como factores que previenen el burnout y sus consecuencias negativas (Schaufeli y Bakker, 2004). En este contexto, ha emergido el concepto de engagement, que hace referencia a la vinculación, la implicación, el compromiso, la pasión, el entusiasmo, el esfuerzo y la energía surgida en el ámbito laboral. Vigor, dedicación y absorción son los tres ejes vertebradores de este constructo: el vigor hace referencia al esfuerzo, energía y persistencia en la labor docente desarrollada a pesar de posibles dificultades que puedan surgir; la dedicación está relacionada con altos niveles de implicación, entusiasmo y reto en las tareas; por último, la absorción está integrada por la capacidad de concentración y de felicidad durante el desempeño laboral. En la investigación disponible se suele admitir que el engagement, asociado a un estado de realización, produce resultados positivos, tanto a nivel individual (crecimiento y desarrollo personal) como a nivel organizacional (calidad del rendimiento). El estado de realización personal derivado del engagement supone la consecución de un estado psicológico de bienestar total laboral, una antítesis del burnout (Bakker, Schaufeli, Leiter y Taris, 2008). Como es sabido, el bienestar está asociado a una autoevaluación global positiva, dentro de un amplio espacio temporal, sobre la propia vida (Hué, 2008). El trabajador engaged tiene un sentimiento de conexión energética y efectiva con su trabajo, percibiéndolo como algo retador.

\section{Evaluación del engagement en el ámbito universitario}

Hay investigaciones relacionadas con el engagement de indudable valor para contextualizar todos los potenciales estudios en este campo (Durán, Extremera y Rey, 2001; Maslash, Schaufeli y Leiter, 2001; Parra, 2007; Seligman y Csikszentmihalyi, 2000). Asimismo, se cuenta con un notable acervo de investigaciones reunidas en torno a la evaluación del profesorado, pero no se ha desarrollado suficientemente la investigación sobre el engagement y sus posibilismos en el profesorado universitario, aunque algunos estudios puedan proporcionarnos pautas evaluativas sobre ciertos aspectos cognitivos y emocionales (Bermejo, 2011; Bermejo y Prieto, 2015; Garga- 
Ilo, 2008; Gargallo, Suárez, Garfella y Fernández, 2011; Galán, González-Galán y Rodríguez-Patrón, 2014; Reyero, 2014). Hallamos aquí un espacio cóncavo de investigación relacionado con la efectividad o "bondad" que este constructo puede reportar al bienestar del docente universitario. Concretamente, encontramos la necesidad de profundizar en la delimitación de algunos indicadores evaluativos del engagement que orienten su racionalidad práctica y teórica.

\section{Definición del problema y delimitación de los objetivos de la investigación}

Las nuevas condiciones laborales y organizativas, insertas en el Espacio Europeo de Educación Superior, el incremento de las exigencias profesionales, la universalización de la educación, la denominada accountability (Conner y Rabovsky, 2011), el escaso reconocimiento social, la falta o disminución de recursos, la carencia o insuficiencia de incentivos y reconocimientos, los desajustes entre desempeños propios de la profesión, etc., configuran escenarios de desgaste emocional en el profesorado universitario que, en no pocos casos, le conducen a una crisis identitaria, sustentada en el desequilibrio provocado por la falta de concordancia entre el proyecto de identidad de cada profesor y la identidad atribuida socialmente, que no resulta fácil afrontar de modo óptimo.

Dudas sobre el quehacer profesional, percepción pesimista del estado de la docencia universitaria, modelos de docencia desfasados y con mínima evolución, poca satisfacción profesional y debilitación de los vínculos con su mundo laboral, serían los principales configuradores de un perfil identitario profesional afectado por el malestar de un docente que se encuentra "quemado" en su profesión, con todo lo que conlleva: alumnos, institución, compañeros, políticas, etc., Ilegando, en no pocas ocasiones, a verse afectada su salud física y emocional. Es sabido que el bienestar emocional es una condición necesaria para la buena actividad educativa. "Hay que sentirse bien para educar bien" (Marchesi, 2007: 141). Pero la componente emocional de bienestar se sustenta básicamente en la consecución de la propia autoestima (Bermejo y Prieto, 2015).

La investigación que planteamos va encaminada a "descubrir" que existen profesores en la universidad que se sienten plenos en su ámbito laboral, que están satisfechos con su profesión y que una combinación acertada de investigación, docencia y pasión por la labor que realizan les conduce a un alto índice de engagement, motor que les ayudará a continuar con su preciada labor educativa, frecuentemente no alejada de la excelencia profesional. Ellos se convierten en foco de atención para el hallazgo de estrategias de afrontamiento del malestar docente y de búsqueda del ansiado bienestar en la profesión.

Una interrogante generadora de otras anejas orienta nuestro estudio: ¿Qué patrones comportamentales caracterizan al profesor engaged? En torno a ella, destacamos las siguientes: ¿Qué significa ser profesor universitario para el docente engaged?, ¿Cómo se siente en el marco universitario actual?; ¿qué dificultades y posibilidades encuentra en su práctica profesional?; ¿es posible elaborar indicadores evaluativos del profesor engaged?

Así, nos hemos planteado los siguientes objetivos: 1) Mostrar causas y repercusiones del malestar y bienestar docente en el ámbito universitario actual, enfatizando 
la relevancia de la dimensión emocional. 2) Matizar los elementos que configuran la identidad del profesorado universitario y su relación con el engagement. Describir dimensiones emocionales y personales asociadas. 3) Elaborar categorías de análisis que coadyuven a una mejor interpretación de los procesos de engagement en el profesor universitario. 4) Delimitar indicadores para la evaluación de la formación de procesos de engagement en el docente universitario.

\section{Método}

La investigación es de carácter cualitativo y tiene como foco de interés principal las construcciones sobre la realidad realizadas por los propios sujetos, objeto del estudio (Flick, 2004). Los modelos de investigación cualitativa tratan de desvelar la riqueza y diversidad de matices, significados e intenciones con que se manifiesta un mismo fenómeno (Álvarez-Gayou, 2005). Desde una perspectiva inductiva se ha trazado el diseño metodológico (Denzin y Lincoln, 2008; Ryan y Bernard, 2003), buscando la información relevante a partir del análisis de casos de la muestra seleccionada (Patton, 2002; Rubin y Rubin, 1995), siguiendo los principios básicos de la metodología biográfico-narrativa (Bolívar, Domingo y Fernández, 2001).

\section{Muestra}

La muestra está compuesta por diez docentes de la Universidad de Sevilla con una dilatada y reconocida trayectoria académica. El muestreo es intencional y se ha seleccionado a los sujetos mediante los criterios de excelencia reconocida (docente e investigadora), de extensa carrera profesional (sólido umbral mínimo de experiencia profesional) y de representatividad epistemológica (incluyéndose todas las ramas de conocimiento actualmente establecidas). La muestra está conformada por seis catedráticos y cuatro profesores titulares (4 mujeres y 6 hombres), con una media de experiencia de 27.9 años. Todos los docentes aceptaron participar voluntariamente en la investigación tras conocer el procedimiento de la misma, lo que les confirió el estatus de "informantes clave" (Goetz y LeCompte, 1988).

\section{Recogida de información}

Se ha recogido información sobre aspectos muy concretos de la vida profesional del docente universitario: identidad profesional, futuro de la profesión y forma de vida. Profundizar en el sentido que tiene para sus vidas el ser docentes, en cómo viven la profesión, qué dedicación le otorgan a la faceta profesional o qué les mantiene vigorosamente en la profesión, entre otros, han sido los principales aspectos considerados.

Hemos considerado apropiada la técnica de la entrevista cualitativa y semiestructurada (Flick, 2004). En la elaboración de la entrevista se realizó un estudio analítico de contenido de la literatura científica existente sobre nuestro problema de investigación del cual se extrajo el conjunto de dimensiones sobre las que versaron las preguntas (descriptivas, verificativas y de contraste) (Callejo, 2009). El guión fue sometido al juicio de diez expertos que arrojaron luz y clarificaron algunas cuestiones 
(Bisquerra, 2004; Hernández, Fernández y Baptista, 2006), quedando definitivamente 24 ítems de los 32 iniciales de que constaba. Los principales ejes vertebradores de la entrevista fueron: 1) identidad profesional; 2) forma de vida; y 3) expectativas. La información recogida fue tratada mediante el programa Aquad 6, específico para el análisis de datos cualitativos, presentados en nuestro caso como textos escritos (transcripciones de las entrevistas realizadas).

Siguiendo las estrategias adecuadas para la gestión de la calidad en la investigación cualitativa (Flick, 2014), se ha procurado utilizar criterios regulativos en el proceso investigador: credibilidad (se hicieron comprobaciones de la información registrada con los participantes), transferibilidad (se procuró realizar la descripción más exhaustiva posible mediante la grabación completa de las entrevistas y la transcripción posterior), dependencia (se conocía detalladamente el perfil de cada informante en función de los criterios de selección muestral) y confirmabilidad (se comprobó por parte de todos los participantes el conjunto de la información correspondiente finalmente elaborada para la fase de análisis).

\section{ANÁLISIS Y DISCUSIÓN DE RESULTADOS}

El análisis de la información registrada se articula en torno a ciertas variables externas e internas que condicionan el engagement (véase Tabla 1). Seguidamente, se muestra según el orden categorial establecido, sin que en nuestro estudio hayamos detectado diferencias destacables entre el profesorado entrevistado desde el punto de vista de alguna variable en particular.

Tabla 1. Variables condicionantes del engagement

\begin{tabular}{|c|c|c|c|}
\hline Categoría & Subcategoría & Descriptores & $\begin{array}{c}\text { Porcentajes } \\
\text { totales }\end{array}$ \\
\hline \multirow{6}{*}{$\begin{array}{l}\text { Identidad } \\
\text { profesional }\end{array}$} & \multirow[b]{2}{*}{ Elección de la profesión } & Vocación & 80 \\
\hline & & $\begin{array}{c}\text { Estabilidad / Inestabilidad } \\
\text { económica y laboral }\end{array}$ & 50 \\
\hline & \multirow{2}{*}{ Satisfacción profesional } & Clima laboral & 80 \\
\hline & & Estilo docente & 80 \\
\hline & \multirow{2}{*}{ Buenas prácticas } & $\begin{array}{l}\text { Aprendizaje efectivo del } \\
\text { alumnado }\end{array}$ & 90 \\
\hline & & $\begin{array}{c}\text { Dimensión práctica y externa } \\
\text { de la formación }\end{array}$ & 70 \\
\hline \multirow{3}{*}{$\begin{array}{l}\text { Forma de } \\
\text { vida }\end{array}$} & \multirow{2}{*}{$\begin{array}{l}\text { Conciliación entre la vida } \\
\text { profesional y la personal }\end{array}$} & Conciliación intraprofesional & 90 \\
\hline & & $\begin{array}{c}\text { Dificultades y posibilidades } \\
\text { de la conciliación }\end{array}$ & 70 \\
\hline & Reconocimiento social & Prestigio social & 100 \\
\hline \multirow{2}{*}{ Expectativas } & Futuro de la profesión & Pesimismo / optimismo & 90 \\
\hline & Selección y formación & Mejora de la formación & 100 \\
\hline
\end{tabular}




\section{Variables condicionantes del engagement}

Confío en las personas, aunque no tanto en el sistema. Éste puede ser mejorado con personas más justas e inteligentes. La profesión será más excelente en la medida en que lo sean los miembros que la integran (Sujeto 5).

Esta visión resulta, desde nuestro enfoque, una condición necesaria, pero no suficiente. El análisis que sigue trata de revelar que junto al saber y a la responsabilidad moral, precisamos partir desde el propio bienestar emocional. Este entramado (bienestar + saber + responsabilidad moral) configura intrincadamente lo que denominamos engagement, fenómeno que podemos incluir entre las "estrategias identitarias", ese margen de maniobra existente para afrontar las crisis y las adversidades del que disponen los sujetos.

\section{Identidad profesional}

\section{Elección de la profesión}

La elección profesional muestra una clara vinculación con nuestro tema objeto de estudio, puesto que el desempeño docente entusiasta está estrechamente relacionado con la vocación, que es recurrentemente esgrimida por el conjunto de la muestra como imantadora de su decisión. El aspecto vocacional parece ser el principal factor de satisfacción y apego a la profesión, siendo uno de los principales motivos que Ileva al docente a ejercer su labor con pasión y de forma comprometida, así lo avala el $80 \%$ de las respuestas emitidas por los entrevistados. La docencia suele ser una dimensión vocacional que destacan los entrevistados, un fuerte aliciente profesional que se ha subrayado también en otros estudios (Guerrero, 2002). A modo de ejemplo, el Sujeto 4 (S4) afirma: "Me apasiona esto (...) estar en la frontera del conocimiento". También S4 y S5 coinciden al respecto. En concreto, S5 añade: "es una labor vocacional, por lo que no tengo que hacer grandes esfuerzos para encontrar la satisfacción".

Las condiciones laborales también han sido recurrentemente referidas. La mitad de la muestra considera que los docentes sufren un fuerte desgaste emocional que repercute en un debilitamiento de su bienestar como consecuencia de las condiciones laborales de inestabilidad y de precariedad (escasez de recursos materiales y humanos, también asociadas a remuneraciones económicas bajas respecto del nivel requerido para la profesión). Todos sufren o han sufrido en algún momento de su carrera profesional descontento con las políticas educativas planteadas, la gestión y administración de la institución o la propia jerarquización y estructura interna de la misma.

Alrededor del $60 \%$ reconoce no haber padecido repercusión física del malestar pero sí conoce a compañeros que la han sufrido, coincidiendo con otras investigaciones al respecto (Martínez-Abascal y Bornas, 1992; Maslash, Schaufeli y Leiter, 2001).

\section{Satisfacción profesional}

El clima laboral puede ser un factor determinante. La capacidad de establecer relaciones humanas óptimas ocupa una posición relevante para los entrevistados, todos coinciden en la importancia de las buenas relaciones entre compañeros y con los 
alumnos, así como el beneficioso reporte emocional que ello conlleva. La creación de un clima agradable de trabajo que permita desarrollar el quehacer profesional con un estilo propio de enseñanza, ha sido muy valorado. Así, S5 nos dice:

esta profesión tiene un gran reto: la autoexigencia, la creación de una forma personal de hacer, el continuo aprendizaje y eso favorece el crecimiento del entusiasmo.

En efecto, la posibilidad de realización del propio estilo educativo facilita la aparición de la proactividad, de la anticipación a las necesidades y problemas propios de su quehacer, buscando soluciones, al tiempo que dispone activa y permanentemente hacia el perfeccionamiento profesional, la concentración en las actividades propias de la profesión y la persistencia ante las adversidades que emergen de la propia práctica. Ahora bien, comúnmente los climas laborales no son todo lo propicios que sería deseable, convirtiéndose en escenarios emocionales tóxicos y generadores de estrés, ansiedad, nerviosismo, enfrentamientos, etc. Para afrontar debidamente estas situaciones, la mayoría de los sujetos entrevistados (80\%) reclama el valor del esfuerzo personal, de la persistencia y energía precisas para la labor y lucha contra la adversidad (en esta misma dirección se sitúa la obra de Amabile y Steve, 2011). Señalan como fuentes principales de alimentación su vocación, la relación educativa con el alumnado, la investigación y el estímulo continuo de la propia formación. El S6 manifiesta:

Creo que la base de todo es la investigación, si las personas no mantienen continuidad investigadora están perdidas...

Se reivindica, por tanto, la mejora desde lo que resulta factible; como ha indicado Bolívar (2012), tal vez deberíamos pensar, antes que en un modelo ideal de universidad perfecta pero imposible, en la importancia de un modelo de universidad centrado en lo que se puede hacer, hic et nunc, a partir de buenas experiencias y propuestas.

\section{Buenas prácticas}

En su cotidianeidad, los docentes encuentran diferentes fuentes motivacionales que les vinculan, aún más, de modo entusiasta con su profesión y que desarrollan en ellos una emocionalidad positiva. La consideración del alumnado como eje principal de atención, aplicando modelos pedagógicos centrados en su aprendizaje efectivo, se ha mostrado relevante para la mayoría (90\%), coincidiendo en determinar la idoneidad de una educación integral en los alumnos que combine el componente intelectual con el moral (Gargallo, 2008; Gargallo et al., 2014), y que reclama sin solución de continuidad una óptima relación pedagógica. Así, S8 expone: "La relación con mis alumnos (...) ha sido siempre mi motor". Del mismo modo, S2 manifiesta "el trato con los alumnos (...) me supone una gran satisfacción".

Las perspectivas cultural y profesional de la enseñanza universitaria se perciben convergentes, coincidiendo con otros trabajos (Tomás, 2006). En este sentido, la mayoría (70\%) reivindica el valor de la dimensión práctica y externa de la formación de los estudiantes como signo del propio bienestar emocional del docente a través de las relaciones educativas establecidas: contactos y transferencias con empresas, prácticas clínicas, etc. 
Forma de vida

Conciliación entre la vida profesional y la personal

La conciliación entre la vida personal y profesional suele ser una de las principales cuestiones que se trataron en la entrevista dada su importancia para el equilibrio emocional. Ser profesor universitario implica una forma de vida, coincidiendo el conjunto de la muestra en manifestar que su ejercicio exige plena absorción, gran exigencia y esfuerzo, y una dedicación permanente, conforme a la evolución misma de la profesión (García-Rodríguez y González-Losada, 2007). "Se es docente todo el día, es un modo de vida", "el ser docente es como un traje que no te puedes quitar", expresan respectivamente S3 y S2. Conciliar la propia vida profesional pasa por armonizar de manera acertada y eficaz distintas actividades principales, que a su vez se despliegan en un amplio haz de nuevas exigencias y desempeños: docencia, investigación, gestión y transferencia del conocimiento. Las dificultades para la conciliación de la actividad profesional con la vida personal no son pequeñas y no siempre se superan (30\% de la muestra), aunque la conciliación entre los distintos ámbitos de la vida se ha señalado por la mayoría como un elemento de bienestar. La búsqueda de esta conciliación parece una consecuencia misma de la pasión por la profesión (Day, 2011). Detengámonos por un momento en las palabras del S7:

Soy docente todo el día, aunque corte aquí a las tres de la tarde, trabajo todas las tardes, leo para investigar, escribo artículos, preparo las clases, etc. La verdad es que está muy relacionada mi vida profesional con la personal.

\section{Reconocimiento social}

La imagen social del docente, por diferentes razones (falta de apoyos institucionales, deficiencias organizativas en la propia profesión, escasa repercusión social de la labor, etc.), se ha devaluado en los últimos años y esta situación genera un desánimo generalizado suscitador de malestar (Hargreaves et al., 2011). El prestigio profesional conlleva el reconocimiento social y, al tiempo, el prestigio social, que incluye reconocimiento social, influye en el prestigio profesional. En este sentido, todos los entrevistados consideran muy importante y beneficioso emocionalmente dicho reconocimiento por la labor que desempeñan. S7 nos afirma: "creo que falta el reconocimiento necesario a la figura del profesor universitario, que en otros países europeos sí tiene". "Hemos de conseguir uniformidad en la enseñanza universitaria tanto en Europa como en España", agrega S1. S2 insiste:

esto está muy difícil, la propia administración está poniendo constantemente todo más complicado, está creando un mal clima entre los docentes, hay mucha exigencia y la docencia no está valorada.

\section{Expectativas}

Futuro de la profesión

El devenir de la profesión suele ser un aspecto que preocupa a la totalidad de los docentes, puesto que de ello depende su estabilidad laboral, económica, social, etc. (Galán, González-Galán y Rodríguez-Patrón, 2014). Al respecto, nos dice S3: "Incertidumbre de no saber cuáles son las reglas del juego (...) reduce la capacidad 
de trabajar". En general, los docentes entrevistados muestran pesimismo respecto del futuro de la profesión (tan sólo un 10\% considera que la situación mejorará), si no se modifican las actuales condiciones sociales y laborales, cambio desde el que se realizan proyecciones optimistas que dejan una puerta abierta a la esperanza. La ya citada inestabilidad institucional, los nuevos planes de estudios, las políticas educativas, los escasos incentivos y reconocimientos docentes, entre otros aspectos, son los más reseñados para alimentar el estado de pesimismo. Afirma S6:

Veo un futuro muy mediocre, la universidad española está llena de mediocridades; se necesita una reforma profunda, pero fruto del consenso.

\section{Selección y formación}

La visión que la totalidad de los entrevistados tiene sobre la docencia está influida por la necesidad de mejorar la selección del profesorado, la cual reportará beneficios a toda la comunidad universitaria. En este sentido, S3 manifiesta: "El sistema actual de acreditación del profesorado habría que cambiarlo para consolidar la selección de los mejores"; y S6 reclama asimismo una modificación de la selección: "La selección del profesorado tiene que cambiar por el bien de la universidad (...) No se selecciona adecuadamente". También, se insiste en la optimización de la formación inicial del profesorado, muy asociada a la formación permanente. En este punto los entrevistados consideran que los docentes deben estar en constante actualización y formación y que, además, esa formación debe ser de calidad y sometida a un proceso de renovación constante, como suele reconocerse internacionalmente (Forest y Altbach, 2007).

\section{Metaevaluación: factores e indicadores valorativos del engagement en el profesorado universitario}

Del análisis categorial hemos efectuado una metaevaluación para extraer los principales factores e indicadores valorativos del engagement (véanse Figuras 1 y 2). Se ha empleado como criterio diferenciador principal el carácter condicionante o conceptual de cada descriptor. En este sentido, se ha podido establecer una serie de factores condicionantes del bienestar vinculados al engagement: estabilidad institucional, condiciones laborales, reconocimiento (prestigio) social y mejora del sistema de formación del profesorado. La estabilidad institucional, delimitada por las políticas educativas, los planes de estudio, la gestión y administración, y la estructura organizativa, constituye una variable condicionante del engagement. Unas condiciones laborales adecuadas donde se atiendan las demandas docentes, mediante la pertinente dotación de recursos materiales y humanos, la disminución de la ratio del alumnado por grupo, así como la consecución de unas relaciones óptimas que coadyuven al establecimiento de un clima laboral agradable, se advierten como otro factor relevante. Con alto contenido emocional, el reconocimiento social de la labor del profesor universitario y su repercusión en el sistema educativo y en el mundo del trabajo, actualmente debilitado, supone un factor condicionante clave del engagement. El cuidado de la imagen social del profesor se halla ligado claramente a dicho factor. Por último, la mejora de la formación del profesorado, inicial y continua, junto 
al perfeccionamiento de los mecanismos de selección, supone un factor determinante para la configuración del paisaje universitario en general y, particularmente, para la promoción y refuerzo del docente engaged.



Figura 1. Factores condicionantes del engagement

Proponemos cinco indicadores evaluativos ligados al ámbito personal del profesorado engaged, en función del análisis realizado: proactividad, perseverancia, dedicación, relaciones humanas óptimas y conciliación entre los distintos ámbitos de la vida. La disposición activa y permanente hacia el perfeccionamiento profesional, anticipándose cuando sea preciso a las necesidades y problemas propios de su quehacer buscando soluciones, la proactividad, se ofrece como un indicador útil vinculado al engagement. La capacidad de superación de dificultades y obstáculos, de perseverancia, de persistencia y energía en la labor, constituye otro indicador válido del engagement. Asimismo, la dedicación entusiasta a la profesión (que no tiene por qué ser idéntica en sus diversas facetas) es garantía de implicación y afrontamiento de desafíos en las tareas que irán sucediéndose. Igualmente, el establecimiento de relaciones humanas satisfactorias en el entorno laboral y fuera de él incide considerablemente en la emocionalidad positiva del docente. En el alumnado se focalizan dichas relaciones satisfactorias, pivotadas sobre la misma vocación universitaria, pero a ellas contribuyen también decisivamente otros agentes (colegas, personal de administración y servicios, personal externo, etc.) que intervienen en el buen desarrollo de la práctica profesional, en la que puede desplegarse la libertad de expresión con naturalidad. Finalmente, la conciliación entre los distintos ámbitos de la vida se ha desvelado como un indicador notorio del proceso de engagement en el docente universitario. Las múltiples exigencias y la diversificación de actividades que componen hoy la profesión reclaman, en primer lugar, una capacidad de conciliación intraprofesional. Junto a ella, la armonía lograda entre la actividad profesional y la vida personal, asociada igualmente a la vocación, se muestra como un elemento de interés para la consideración del engagement. Tal conciliación denota una sólida madurez emocional desde la que resulta más fácil vivir la profesión en plenitud. En la Figura 2 puede observarse gráficamente el entramado relacional de los indicadores expuestos. 




Figura. 2. Indicadores evaluativos del docente engaged

\section{Conclusiones}

La evolución de la sociedad y de la economía hoy, junto a los cambios propuestos en el nuevo contexto internacional universitario (específicamente, europeo), han incrementado las exigencias en entornos de mayor complejidad para el ejercicio profesional. Estas nuevas condiciones afectan a su propia identidad, sufriendo a menudo un desgaste emocional difícilmente superable. Frente al problema del malestar existente, se ha ido proponiendo una diversidad de estrategias, entre las que ocupa un lugar destacado el fenómeno del engagement, vinculado a las estrategias identitarias y asociado al bienestar profesional. En esta línea, es preciso explorar los principales condicionantes externos del engagement en el profesorado y tratar de profundizar en aquellos elementos que conforman, en el ámbito personal, los resortes que generan la dedicación entusiasta, el vigor necesario para perseverar y la capacidad de disfrute durante el desempeño.

Nuestro estudio, al margen de revelar ciertas condiciones sociolaborales que han ido emergiendo análogamente en la investigación conocida, refleja, después del análisis de los significados, intenciones, percepciones y sentimientos actuales del profesorado entrevistado acerca de la delimitación de lo que es y deber ser la profesión, una serie de cinco indicadores descriptivos del profesor engaged, que encierran una virtualidad: indicar áreas específicas de indagación evaluativa. Al tiempo, albergan una potencialidad formativa, si pensamos en el diseño y desarrollo de programas dirigidos a establecer posibles "anclajes" del engagement en el profesorado. 
Aunar el equilibrio emocional y profesional en una relativa unidad mental y discursiva, capaz de conciliar sus diversas actividades profesionales y éstas con su vida personal, parece ser un eje sobre el cual el profesor puede desarrollar su labor con compromiso, vigor y pasión. Este proceso halla, asimismo, un punto de referencia elemental en el alumnado, que precisamente es centro de interés patente en el nuevo contexto sociopolítico, abriéndose la posibilidad de instalación de una nueva cultura profesional, acaso más propicia, si se acierta con su aplicación, para la generación de procesos de engagement. Del estudio realizado emana enérgicamente la idea de vocación universitaria, vinculada a procesos relacionados con las capacidades de dedicación, tenacidad y resiliencia, así como de goce con el desempeño profesional. Al mismo tiempo, el hallazgo de un estilo educativo propio, armónico entre la identidad proyectada y la reconocida o asignada, también ha aflorado como un indicador que deberíamos considerar.

Posiblemente, se precisa insistir en estudios que aboguen por favorecer la identificación de los profesores, en tanto que personas, con sus entornos profesionales, situándolos ante la posibilidad de expresar sus capacidades específicas como miembros de la comunidad universitaria, suscitando aquellos elementos potenciadores que les predispongan al hallazgo del engagement, siempre un acontecimiento individual, encarnado en la figura concreta de un docente singular en una circunstancia determinada. Estos procesos parecen requerir la superación de la disyuntiva entre una lógica funcional, relacionada con la adaptación a las normas existentes, y una lógica existencial, ligada a las demandas de la experiencia real. En este sentido, cabe profundizar en posibles abstracciones diferenciadoras entre los "perfiles de engagement": investigador, docente, investigador y docente... Asimismo, convendría ahondar en posibles caracterizaciones específicas según la pertenencia a distintas ramas del conocimiento, a diferente género o a distintos tramos de edad. Por otra parte, se precisa contrastar nuestros resultados con otros estudios cualitativos en la misma línea, que traten de profundizar en el perfil genérico del profesor engaged y en sus posibilidades evaluativas.

\section{REFERENCIAS BIBLIOGRÁFICAS}

Álvarez-Gayou, J. L. (2005). Cómo hacer investigación cualitativa. Fundamentos y metodología. México: Paidós.

Amabile, T. y Steve, J. (2011). The Progress Principle: Using Small Wins to Ignite Joy. Engagement, and Creativity at Work. Harvard: Harvard Business Press.

Amador, R., Rodríguez, C., Serrano, J., Olvera, J. A. y Martínez, S. (2014). Estrés y burnout en docentes de educación media superior. Revista Electrónica de Medicina, Salud y Sociedad, 4(2), 119-141.

Augusto-Landa, J. M., López-Zafra, E., Martínez de Antoñana, R. y Pulido-Martos, M. (2006). Perceived emotional intelligence and life satisfaction among university teachers. Psicothema, 18(1), 152-157.

Bakker, A. B., Schaufeli, W. B., Leiter, M. P. y Taris, T. W. (2008). Work engagement: An emerging concept in occupational health psychology. Work \& Stress, 22(3), 187-200. DOI: http://doi.org/10.1080/02678370802393649. 
Barraca, J. (2010). Emociones negativas en el profesorado universitario. Burnout, estrés laboral y mobbing, EduPsykhé. Revista de Psicología y Educación, 9(1), 85-99.

Beck, U. y Beck-Gernsheim, E. (2003). La individualización: el individualismo institucionalizado y sus consecuencias sociales y políticas. Barcelona: Paidós.

Bermejo, L. (2011). Demandas y recursos en el bienestar docente. Estrategias de afrontamiento y su relación con el burnout y el engagement en profesores. Tesis doctoral, Facultad de Ciencias Humanas y Sociales, Universidad Pontifica de Comillas.

Bermejo, L. y Prieto, M. (2015). Bienestar docente. Del síndrome de burnout al engagement en profesores. Madrid: Publicia.

Bisquerra, R. (coord.) (2004). Metodología de la investigación educativa. Madrid: La Muralla.

Bolívar, A. (2012). La cultura universitaria: contextos y metas actuales para una identidad académica y pedagógica innovadora. En A. de la Herrán y J. Paredes (coords.), Promover el cambio pedagógico en la universidad (pp. 41-56). Madrid: Pirámide.

Bolívar, A., Domingo, J. y Fernández, M. (2001). La investigación biográfico-narrativa en educación. Madrid: La Muralla.

Bresó, E., Salanova, M., Schaufeli, W. y Nogareda, C. (2005). NTP 732 Síndrome de estar quemado por el trabajo "Burnout" III: Instrumento de Medición. Ministerio de Trabajo y Asuntos Sociales de España. Consultado enero 2012 en "Construyendo inéditos viables" Primer Congreso Internacional de Educación 2114 Área Temática 8: Educación y salud. Recuperado de http://www.insht.es/InshtWeb/Contenidos/ Documentacion/FichasTecnicas/NTP/Fi cheros/701a750/ntp_732.pdf.

Caballero, C., Hederich, C. y Palacio, J. (2010). El burnout académico: delimitación del síndrome y factores asociados con su aparición. Revista Latinoamericana de Psicología, 42(1), 131-146.

Caballero, K. (2009). Construcción y desarrollo de la identidad profesional del profesorado universitario. Tesis Doctoral. Universidad de Granada.

Caballero, K. y Bolívar, A. (2015). El profesorado universitario como docente: hacia una identidad profesional que integre docencia e investigación. Revista de Docencia Universitaria, 13(1), 57-77.

Callejo, J. (coord.) (2009). Introducción a las técnicas de investigación social. Madrid: Editorial Universitaria Ramón Areces-UNED.

Cantón, I., Valle, R. E. y Arias, A. R. (2008). Calidad de la docencia universitaria: procesos clave. Educatio Siglo XXI, 26, 121-160.

Conner, T. y Rabovsky, T. (2011). Accountability, Affordabililty, Access: A Review of the Recent Trends in Higher Education Policy Research. Policy Studies Journal, 39(1), 93-112.

Day, C. (2011). Pasión por enseñar. La identidad personal y profesional del docente y sus valores. Madrid: Narcea.

Denzin, N. y Lincoln, Y. (ed.) (2008). Strategies of qualitative inquiry. California: Sage Publications.

Dubet, F. (2006). El declive de la institución (profesiones, sujetos e individuos en la modernidad). Barcelona: Gedisa. 
Durán, A., Extremera, N. y Rey, L. (2001). Burnout en profesionales de la enseñanza: un estudio en Educación primaria, secundaria y superior. Revista de Psicología del Trabajo y de las Organizaciones, 17, 45-62.

Flick, U. (2004). Introducción a la investigación cualitativa. Madrid: Morata.

Flick, U. (2014). La gestión de la calidad en la Investigación Cualitativa. Madrid: Morata.

Forest, J. y Altbach, Ph. (eds.) (2007). International Handbook of Higher Education. Dordrecht: Springer. Book Series, vol. 18.

Galán, A., González-Galán, M. A. y Rodríguez-Patrón, P. (2014). La evaluación del profesorado universitario en España. Sistema nacional y divergencias territoriales. Revista de Educación, 366, 136-164. DOl: http://doi.org/10.4438/1988-592XRE-2014-366-279.

García Carrasco, J. y Bernal, A. (2008). Institución y decepción. La salubridad institucional y la práctica docente. Revista española de pedagogía, 66(241), 405-423.

García-Rodríguez, M. P. y González-Losada, S. (2007). El perfil del profesorado universitario: un profesional en evolución constante. XXI, Revista de Educación, 9, 181-205.

Gargallo, B. (2008). Estilos de docencia y evaluación de los profesores universitarios y su influencia sobre los modos de aprender de sus estudiantes. Revista Española de Pedagogía, 66(241), 425-446.

Gargallo, B. et al. (2014). Metodología centrada en el aprendizaje. Su impacto en las estrategias de aprendizaje y en el rendimiento académico de los estudiantes universitarios. Revista Española de Pedagogía, 72(259), 415-435.

Gargallo, B., Suárez, J. M., Garfella, P. R. y Fernández, A. (2011). El cuestionario CEMEDEPU: un instrumento para la evaluación de la metodología docente y evaluativa de los profesores universitarios. ESE: Estudios sobre educación, 21, 9-40.

Giddens, A. (2000). Un mundo desbocado. Los efectos de la globalización en nuestras vidas. Madrid: Taurus.

Goetz, J. P. y LeCompte, M. D. (1988). Etnografía y diseño cualitativo en investigación educativa. Madrid: Morata.

Guerrero, E. (2002). Un trabajo de investigación sobre estrés y "burnout" en el profesorado universitario. Revista de Educación, 21, 97-118.

Hargreaves, A. (2003). Enseñar en la sociedad del conocimiento. Barcelona: Octaedro.

Hargreaves, A. et al. (2011). Introduction. Second International Handbook of Educational Change (pp. XI-XXI). Dordrecht: Springer.

Hernández, R., Fernández, C., y Baptista, P. (2006). Metodología de la investigación (4⿳亠丷厂 ed.) México: Mc-Graw Hill.

Hué, C. (2008). Bienestar docente y pensamiento emocional. Madrid: Wolters Kluwer.

Ibarra, E., Armenta, M. y Jacobo, H. M. (2014). Autoconcepto, estrategias de afrontamiento y desempeño docente profesional. Estudio comparativo en profesores que trabajan en contextos adversos. Profesorado. Revista de Currículum y Formación del Profesorado, 18(1), 223-239.

Marchesi, A. (2007). Sobre el bienestar de los docentes: Competencias, emociones y valores. Madrid: Alianza. 
Martinez Abascal, M. A. y Bornas i Agustí, X. (1992). Malestar docente, atribuciones y desamparo aprendiendo: un estudio correlacional. Revista Española de Pedagogía, 50(193), 563-592.

Maslash, C., Schaufeli, W. B. y Leiter, M. P. (2001). Job burnout. Annual Review of Psychology, 52, 397-422.

Matud, M. P., García, M. A. y Matud, M. J. (2006). Estrés y malestar en el profesorado. International Journal of Psychology and Psychological Therapy, 6(1), 63-76.

Parra, M. (2007). La salud de los docentes: revisión de líneas emergentes en la investigación e intervención. Santiago: UNESCO/OREALC.

Patton, M. Q. (2002). Qualitative research \& Evaluation methods (3 ${ }^{\mathrm{a}}$ ed.) Thousand Oaks, CA: Sage.

Reyero, D. (2014). La excelencia docente universitaria. Análisis y propuestas para una mejor formación del profesorado universitario. Educación XXI, 17(2), 125-143.

Rubin, H. J. y Rubin, I. S. (1995). Qualitative interviewing. The art of hearing data. Thousand Oaks, CA: Sage.

Ryan, G. W. y Bernard, H. R. (2003). Data management and analysis methods. En N. K. Denzin y Y.S. Lincoln (eds.) Collecting and interpreting qualitative materials (2 ${ }^{\mathrm{a}}$ ed.) (pp. 259-309). Thousand Oaks, CA: Sage.

Salanova, M. y Schaufeli, W. B. (2009). El engagement de los empleados. Cuando el trabajo se convierte en pasión. Madrid: Alianza.

Sánchez, D., March, M. X. y Ballester, L. (2015). Malestar social y malestar docente: una investigación sobre el síndrome de desgaste profesional burnout y su incidencia socioeducativa. Aula, 21, 245-257. DOI: http://doi.org/10.14201/aula201521245257.

Schaufeli, W. B. y Bakker, A. (2004). Job Demands, Job Resources and their Relationship with Burnout and Engagement: a Multi-Sample Stud. Journal of Organizational Behaviour, 25, 293-315.

Seligman, M. E. P. y Csikszentmihalyi, M. (2000). Positive psychology: An introduction. American Psychologist, 55, 5-14.

Sennett, R. (2000). La corrosión del carácter. Las consecuencias personales del trabajo en el nuevo capitalismo. Barcelona: Anagrama.

Tomás, M. (coord.) (2006). Reconstruir la universidad a través del cambio cultural. Barcelona: UAB.

Touraine, A. (2005). Un nuevo paradigma para comprender el mundo de hoy. Barcelona: Paidós.

Vázquez, C. y Hervás, G. (2009). La ciencia del bienestar: Fundamentos de una psicología positiva. Madrid: Alianza.

Zembylas, M. (2003). Emotions and teacher identity: a poststructural perspective. Teachers and Teaching: Theory and Practice, 9(3), 213-238. 